\title{
TURBULENCE IN DISKS AND LABORATORY EXPERIMENTS: THE CONTRIBUTION OF JEAN-PAUL ZAHN
}

\author{
B. Dubrulle ${ }^{1}$
}

\begin{abstract}
The activity of Jean-Paul on disks does represent only a small fraction of his total list of publications. It was however very important to its eyes. Beyond his important scientific results on the topic that I summarized, this activity launched a totally new area in the domain of astrophysics: the dedicated laboratory experiments.
\end{abstract}

\section{Introduction}

Jean-Paul Zahn directed my PhD thesis from September 1988 to December 1990. The topics of my PhD was "Instabilities and turbulence in accretion disks". The way how Jean-Paul presented me this topic-which he had just started to study-was as follow: accretion disks are quite frequent in the Universe. They exist around stars, black hole or whenever there is some massive object that is surrounded by some matters. Without any diffusive or mixing process, the matter in the accretion disk would just spin around the central object following a Keplerian law, and no matter will ever reach the central object. We know however from observation that there is some accretion of matter onto the central object, because when it occurs, it produces an energy release that can be detected by telescopes. A natural diffusive process would be the molecular fraction of the gas atoms. However, it has been long ago realized that molecular diffusivity is too weak to produce observed accretion rates. That is why, Zeldovich, then Shakura and Sunyaev suggested that the disk are turbulent, so that the transport processes are governed by turbulent eddies, resulting in a much higher effective viscosity. There is however a mystery: a Keplerian flow is linearly stable with respect to infinitesimal perturbations. So the question is: how can it become turbulent? The goal of my thesis was then first to understand how a Keplerian disk can become turbulent, and then compute the corresponding effective viscosity. The way Jean-Paul presented it sounds really attractive for a young physicist like me. I was of course to naive to understand

${ }^{1}$ SPEC, CEA, CNRS, Université Paris-Saclay, CEA Saclay 91191 Gif-sur-Yvette Cedex, France 
how difficult this topic was, but maybe that even if I had known, I would still have accepted this subject, since Jean-Paul was quite enthousiastic and so charismatic. Moreover, he had the beginning of a clue to solve the problem.

A few years ago, he had indeed worked with his old friends Juri Toomre, Edward Speigel and Douglas Gough on the Poiseuille flow (Zahn et al. 1974), the flow created by a pressure gradient in between two plates. Under certain conditions, this flow is stable to infinitesimal perturbations, but can be destabilized by well chosen finite amplitude perturbations. A pictural description of what is going on is the following: imagine the "laminar non-turbulent" corresponds to a state which is at the bottom of a potential well. Further away, in another valley, lies a turbulent state. In between the two, there is a mountain, with an amplitude that may depend on the flow conditions (for example on the Reynolds number). Now, imagine that your flow state is a little ball travelling in this energy landscape. If you start it in the laminar valley, and only excite it with very small (infinitesimal) perturbation, you can never give it enough impulse to jump across the mountain that separates the laminar from the turbulent valley: whatever infinitesimal small impulse you kick, the ball bounces back to the laminar valley. The flow is linearly stable. Now, imagine you kick the ball with a finite amplitude perturbation, with an energy high enough to cross the mountain. Now the ball can now bounce from the laminar to the turbulent state. The amplitude of the kick is not necessarily very large: if the height of the mountain varies like $R e^{-\alpha}, \alpha=O(1)$, then when the Reynolds number is very large, the kick to provide may be very small! So even if the flow is linearly stable, at very large number, any perturbation will be large enough to cross the mountain, and the flow will be turbulent.

Jean-Paul had from the very start the intuition that such picture could apply to astrophysical disks since their Reynolds number is very large. The main problem was to compute the law of finite amplitude perturbation, i.e. the height of the mountain for Keplerian disks. As a starter, Jean-Paul suggested to me to ignore rotation, and to focus on the shear part of the Keplerian flow, that is to study the much simpler case of the linear shear flow, named the plane Couette flow.

\section{Finite amplitude instabilities in shear flows}

Even this simple case was quite hard to solve. We succeeded in finding a law for the finite amplitude by considering a perturbation under the shape of a "defect" in the mean flow profile and predicted that the amplitude of the perturbation needed to destabilize the plane Couette flow was $A_{t h} \sim R e^{-1 / 3}$ (Dubrulle \& Zahn 1991). This prediction was then verified experimentally by two researchers from the CEA Olivier Dauchot and François Daviaud, who found $A_{\text {exp }} \sim R e^{-0.4 \pm 0.1}$ (Dauchot \& Daviaud 1995). Such a law is sufficiently rapidly decaying at infinity to allow for a wide range of possible perturbations to destabilize the flow. Moreover, Jean-Paul has another idea that may help trigger the instability from an arbitrary perturbation: the scenario of bypass transition via algebraic transient growth (Chagelishvili et al. 2003; Tevzadze et al. 2003). The idea is that in a shear flow that is linearly stable, infinitesimal perturbation do not grow 
exponentially but algebraically, because of he non-normality of operator associated with the linearized equations of motions. This is a rather slow growth, but to achieve instability, one only has to let the perturbation grow until it achieves the $A \sim R e^{-1 / 3}$ law, until one may expect the flow to be destabilized. Therefore, the combination of both mechanism may represent a very efficient way to destabilize any linearly stable shear flow.

\section{From the universe to the laboratory}

This finding was actually not sufficient to explain the turbulence in accretion disks, because there is another major ingredient in Keplerian disk: the rotation. Because of its stabilizing role, this new player may interfere with the finite amplitude instability and makes the turbulence much harder to reach. Jean-Paul was of course aware of that, and since 1996, he started re-examine old experiments about rotating shear flows, to try to understand the crucial role of rotation in the instability. He was able to exhume a very old paper by Wendt (1933) that dealt with this problem, but had fallen into oblivion because it was written all in German. Jean-Paul, a native of Alsace, had a very good knowledge of German, and he was able to translate for himself (and others!) the paper. According to Wendt's finding, they are two different case to be considered, depending on the sign of the rotation $(\Omega)$ with respect to the shear $(S)$ : cyclonic (shear and rotation have the same size) or anti-cyclonic. This can be quantified through a single number, the rotation number $R_{\Omega}=2 \Omega / S$. A rotating shear flow is linearly stable for $-1 \leq R_{\Omega} \leq 0$. A Keplerian flow corresponds to $R_{\Omega}=-4 / 3$ and is therefore linearly stable. However, the experimental study of Wendt shows that in both cases $R_{\Omega} \leq-1$ and $R_{\Omega} \geq 0$, the flow becomes turbulent for large enough Reynolds number. Jean-Paul hired a new PhD, Denis Richard, to investigate this. After a careful study of Wendt's paper, they were able to deduce a shape of the turbulent viscosity from Wendt's measurement. They showed that the characteristic length and time scale involved were the local radius $r$ and the shear rate $S=|d \Omega / d r|$, so that the turbulent viscosity writes $\nu_{t}=\beta r^{3} S$ with $\beta$ drawn from experimental measurements as $\beta=1.5 \pm 0.510^{-5}$ (Richard \& Zahn 1999). So, by using these old experiments, Jean-Paul was able not only to prove that disks can be turbulent, but also to compute the efficiency of such turbulence. This prescription was tested numerically via a collaboration with Jean-Marc Huré (Huré et al. 2001) and was found to be suitable for all three families of known systems: in young stellar objects, evolved binary stars and Active Galactic Nuclei discs. Using realistic opacities and equation of state, they demonstrate that these discs are thermally unstable in the temperature domain where hydrogen recombines, when they are optically thick, and this could lead to limit cycle behavior. Radiation pressure dominated regions are thermally stable, in contrast with alpha -discs. This results in a fully stable solution for the innermost parts of AGN discs.

Jean-Paul was however not fully satisfied with this result because he thought that Wendt's experiment should be redone using our modern means, to get more precision and a better feeling of what is going on. He then convinced the two 
researchers of CEA, O. Dauchot and F. Daviaud, to build an astrophysicallydedicated experiment, i.e. a Taylor-Couette flow especially tailored to reproduce the essential features of an accretion disks. This involved modifications of experimental set-ups that were not common in the experimental community. Jean-Paul took a special care in the designing the upper and lower boundary conditions: he was truly aware that due to upper and lower boundary, an large scale Ekman circulation could settle into the flow and modify the mean flow pattern and the instability properties. He then convinced the experimentalist to use rotating rings at the top and the bottom, to decrease the Ekman pumping. In the Saclay experiment, they used 2 rings. In the latter much more elaborate Princeton experiment, they used up to 5 rings. Once the experiment was built, it was then ran and analyzed in collaboration with D. Richard and myself. This new experiment essentially confirmed the older results by Wendt: a Keplerian shear flow can become turbulent at large enough Reynolds number. The prescription for the viscosity was generalized, in order to apply to any type of flow, and not only to Keplerian flow (Dubrulle et al. 2005). It was found to provide an the effective accretion rate, as a function of the disk characteristic parameters (orbiting velocity, temperature and density) corresponding to and $\alpha$ parameter $2^{-4} \leq \alpha \leq 2^{-2}$. This value is in good agreement with values derived in the solar protoplanetary nebula from chemical composition of comets and planetary atmospheres (Dubrulle 1993; Drouart et al. 1999; Mousis et al. 2000).

\section{Instability through interplay between shear and stratification}

The non-linear instability in the Taylor-Couette system results from a competition between shear and rotation. In the early 1990 (Balbus \& Hawley 1991) exhumed some old work by Velikhov and Chandrasekhar to show that if one adds a vertical magnetic field to the problem, it becomes then much easier to destabilize a rotating shear flow, including the Keplerian ones: the flow can now becomes linearly unstable to infinitesimal perturbations. They named this instability the magnetorotationnal instability (MRI) As already recognized by Chandraskhar, this is a puzzling fact: it is traditionally thought that magnetic field has a "stabilizing influence" on the flows, because perhaps of its "bi-dimensionalization effect": it damps velocity fluctuations in the direction perpendicular to the magnetic field. Indeed, in some classical instability problem such as Rayleigh-Benard convection, the addition of a magnetic field makes the critical Rayleigh number for instability larger. So loosely speaking, it seems here instead that adding a "stabilizing" effect on a stable flow makes it more unstable!

One may then wonder whether the addition of another stabilizing effect to a Keplerian shear flow would not produce the same miraculous effect. Indeed, there is at least one more instance, and Jean-Paul worked on it: it is the case where a vertical stable stratification is added onto the mean Keplerian flow. In that case, exactly like in the MRI case, the Keplerian flow can become unstable to linear infinitesimal small perturbations (Dubrulle et al. 2005). By analogy, this instability was named Strato-Rotationnal Instability (SRI). Quite remarkably, the predicted instability was then searched and detected in a dedicated laboratory 
experiment by Lebars and Legal (Le Bars \& Le Gal 2006). So, it is another example of astrophysical motivated questions providing incentive for new laboratory experiments.

\section{Conclusion}

The activity of Jean-Paul on disks does represent only a small fraction of his total list of publications. It was however very important to its eyes, and until the end of his career, he continued to be willing to discuss related issues and new results in this topic. Beyond his important scientific result on the topic, this activity launched a totally new area in the domain of astrophysics: the dedicated laboratory experiments. Indeed, Jean-Paul, with his bright vision of science had foreseen that laboratory experiments could be a nice alternative to numerical simulations, to advance our knowledge: they can often reach parameter values that are much closer to astrophysical ones than those achieved in numerical simulations. Of course, laboratory experiments cannot fully reproduce the whole complexity of an astrophysical object, and they have to be used with caution, to study well focused and identified problems. Since the pioneering works of Jean-Paul, the use of laboratory experiments have now diffused to the field of neutron stars or supernovae. No doubt that it is only a beginning, and that many other will follow the road from the Universe to the laboratory opened by Jean-Paul!

\section{References}

Balbus, S.A., \& Hawley, J.F., 1991, Astrophys. J., 376, 223

Chagelishvili, G.D., Zahn, J.-P., Tevzadze, A.G., \& Lominadze, J.G., 2003, Astron. Astrophys., 402, 401

Dauchot, O, \& Daviaud, F., 1995, Phys. Fluids, 7, 335

Drouart, A., Dubrulle, B., Gautier, D., \& Robert, F., 1999, Icarus, 140, 129

Dubrulle, B., 1993, Icarus, 106, 59

Dubrulle, B., Dauchot, O., Daviaud, F., Longaretti, P.-Y., Richard, D., \& Zahn, J.-P., 2005, Phys. Fluids, 17, 095103-095103-19

Dubrulle, B., Marié, L., Normand, Ch., Richard, D., Hersant, F., \& Zahn, J.-P., 2005, Astron. Astrophys., 429, 1

Dubrulle B., Zahn J.P., 1991, J. Fluid Mech., 231, 561

Hersant, F., Dubrulle, B. \& Huré, J-M., 2005, Astron. Astrophs., 429, 531

Huré, J.-M., Richard, D., \& Zahn, J.-P., 2001, Astron. Astrophys., 367, 1087

J.-P. Zahn, J. Toomre, E.A., 1974, Spiegel, \& D.O. Gough, J. Fluid Mech., 64, 319

Le Bars, M., \& Le Gal, P., 2006, Phys. Rev. Lett., 99, 064502

Mousis, O. Gautier, O., Bockelee-Morvan, D., Robert F., Dubrulle, B., \& Drouart, A., 2000, Constraints on the formation of comets from D/H ratios measured in $\mathrm{H} 2 \mathrm{O}$ and HCN Icarus, 148, 513

Richard, D., \& Zahn, J-P., 1999, Astron. Astrophys., 347, 734

Tevzadze, A.G., Chagelishvili, G.D., Zahn, J.-P., Chanishvili, R.G., \& Lominadze, J.G., 2003, Astron. Astrophys., 407, 779

Wendt, F., 1933, Arch. Appl. Mech., 4, 577595 